\title{
ESTIMATION OF THE SCALE PARAMETER OF GAMMA MODEL IN THE PRESENCE OF OUTLIER OBSERVATIONS
}

\author{
M.E. GHITANY \\ Department of Mathematics \\ University of Saskatchewan \\ Saskatoon, Saskatchewan \\ Canada, S7N OWO \\ and \\ Department of Mathematics \\ University of Western Auscralia \\ Nedlands, WA 6009 \\ Australia
}

(Recelved August 15, 1988)

ABSTRACT. This paper considers the Bayestan point estimation of the scale parameter for a two-parameter gamma life-testing model in presence of several outlier observations in the data. The Bayesian analysis is carried out under the assumption of squared error loss function and fixed or random shape parameter.

KEY WORDS AND PIRASES. Scale parameter, gamma distribution, squared error loss function, noninformative prior, Bayes estimate, incomplete gamma function, modified Bessel function of the third kind.

1980 AMS SUBJECT CLASSIFICATION CODES. $62 \mathrm{C10}$.

1. INTRODUCTION.

Bayesian estimation problems taking into account possible extension of the parameter set of a basic probability model to include contamination components, called outliers, have been considered for some time. An excellent review for the Bayesian approaches to outliers can be found in Barnett and Lew 1s [1].

In particular, the published literature dealing with the Bayesian point estimation for the scale parameter of most well known 1 ife-testing models in presence of several outlier observations in the data, is limited.

Sinha [2] seems to be the first to consider a fuller Bayestan analysis in the exponential distribution when a single outlier observation may be present in the data. Employing three possible families of prior Aistributions (inverted gamma, uniform, noninformative), he provided the Bayes estimator for the scale parameter under each of these priors.

Lingappaiah [3] generalizes the corresponding estimation problem of Sinha [2] by considering the generalized gamma distribution when several outliers may be present in 
the data. Accordingly, he derived the Bayes estimator of the scale parameter for each of che exponential, gamma and Weibul1 distributions. However, assuming fixed shape parameter, he only employed one particular member of the inverted gamma prior family for the scale parameter.

It should be mentioned here that in both of the above two papers, the authors employed beta prior family to each parameter causing the contamination in the data. Only, Sinha [2], briefly discussed the robustness of the posterior distribution of the scale parameter when the parameter causing the contamination is $\mathrm{f} 1 \mathrm{xed}$.

Also, Lingappaiah [4] studies the effect of a single outlier observation on the Bayesian estimation of the parameters of normal life-testing distribution model.

In this paper, we consider the following point estimation problem. Suppose an observed sample $\left(x_{1}, x_{2}, \ldots, x_{n}\right)$ in such that $n-k$ observations are from the gamma family with p.d.f

$$
f(x ; \sigma)=\frac{1}{\Gamma(\mu) \sigma^{\mu}} \exp [-x / \sigma] x^{\mu-1}, \quad x>0, \sigma, \mu>0,
$$

where $\mu$ is the shape parameter, while $k$ observations are from different families with p.d.f's $f\left(x ; \sigma / \alpha_{r}\right), 0<\alpha_{r}<1, r=1,2, \ldots, k$. Assume that the parameters $\alpha^{\prime} s$ are fixed and the set of $k$ outliers is likely to be any set of $k$ observations from the sample. Under squared error loss function, the main problem here is to find a closed form expression for the Bayes estimator of the scale parameter $\sigma$ when the shape parameter $\mu$ is either a fixed real number or a random variable, respectively. For fixed shape parameter $\mu$ the following prior familles of distributions are considered:

(i) Inverted Gamma family

$$
g(\sigma)=\frac{a^{b}}{\Gamma(b)} \exp [-a / \sigma] \quad \sigma^{-(b+1)}, \quad \sigma>0, \quad a, b>0,
$$

(This is a natural conjugate prior for $\sigma$ (Ralffa [5]));

(ii) Uniform family

$$
g(\sigma)=\frac{1}{a_{2}-a_{1}}, \quad a_{1}<\sigma<a_{2},
$$

(iii) Exponential famfly

$$
g(\sigma)=\lambda^{-1} \exp [-\sigma / \lambda], \quad \sigma>0, \quad \lambda>0 ;
$$

(1v) Noninformative family

$$
g(\sigma)=\sigma^{-c}, \quad \sigma>0, \quad c>0 .
$$

For random shape parameter $\mu$, we consider a mixture of discrete prior and a conditional inverted gamma prior distribution on $\mu$ and $\sigma$, respectively, as follows:

$$
\operatorname{Pr}\left\{\mu=\mu_{j}\right\}=p_{j}, \quad j=1,2, \ldots, m, \quad \mu_{j}>0,
$$




$$
g\left(\sigma \mid \mu=\mu_{j}\right)=\frac{\mu_{j}^{\nu_{j}}}{\Gamma\left(\nu_{j}\right)} \exp \left\{-\mu_{j} / \sigma\right\} \sigma^{-\left(\nu_{j}+1\right)}, \sigma>0, \mu_{j}, \nu_{j}>0,
$$

(This is a natural conjugate prior for $(\mu, \sigma)$ (Raiffa [5])).

Note that all the above priors were considered, partially or totally, in the work of Sinha [2], Lingappaiah [3,4], Bhattacharya [6], Canavos and Tsokos [7], and Lwin and Singh $[8]$.

In the subsequent sections we will derive the posterior mean, $1 . e$. the Bayes estimator, and variance of the scale parameter $\sigma$ under each of the above priors, respectively.

\section{ESTIMATION OF $\sigma$ WHEN $\mu$ IS FIXED}

To work out the Bayes estimator of $\sigma$, note that the likelihood function can be expressed as

$$
L(\sigma) \propto \sum_{\underline{i}} \prod_{j+1}^{n} f\left(x_{j} ; \sigma\right) \prod_{r=1}^{k} \frac{f\left(x_{1} ; \sigma / \alpha_{r}\right)}{f\left(x_{j} ; \sigma\right)},
$$

where the summation denotes the $k-f o l d$ sums over the components of the vector $\underline{1} \equiv$ $\left(i_{1}, i_{2}, \ldots, i_{k}\right)$ such that $1<i_{1} \neq i_{2} \neq \ldots \neq i_{k}<n$. This likelihood function must satisfy the following relation:

$$
L(\sigma) \propto \sum_{\underline{1}} \exp \left[-w_{\underline{1}}(\underline{\alpha}) / \sigma\right] \sigma^{-n},
$$

where $\underline{\alpha} \equiv\left(\alpha_{1}, \alpha_{2}, \ldots, q_{k}\right)$ and

$$
w_{1}(\underline{\alpha})=\bar{n} \bar{x}-\sum_{r=1}^{k}\left(1-\alpha_{r}\right) x_{1},
$$

where $\bar{x}$ is the sample mean.

Now, the likelihood function and the prior density of $\sigma$ are used to obtain the posterior density. That is, according to Bayes' theorem the posterior density of $\sigma$ is given by

$$
\pi(\sigma)=\frac{L(\sigma) g(\sigma)}{\int_{\Omega} L(\sigma) g(\sigma) d \sigma},
$$

where $\Omega$ is the parameter space containing $\sigma$. Consequently, we can find the posterior mean and variance for $\sigma$.

Respectively considering the priors (1.2), (1.3), (1.4) and (1.5), the posterior densities of $\sigma$ are as follows:

where

$$
\pi(\sigma)=A^{-1}(n \mu, \underline{\alpha}, a, b) \sum_{\underline{1}}^{\sum} \exp \left[-\left\{a+w_{\underline{1}}(\underline{\alpha})\right\} / \sigma\right] \quad \sigma^{-(n \mu+b+1)},
$$

$$
A(n \mu, \underline{\alpha}, a, b)=\Gamma(n \mu+b) \sum_{\underline{1}}^{\sum}\left[a+w_{1}(\underline{\alpha})\right]^{-(n \mu+b)} ;
$$


where

$$
B\left(n \mu, a_{1}, a_{2}\right)=\sum_{\underline{1}}\left[w_{1}(\underline{\alpha})\right]^{-(n \mu-1)} \gamma^{\star}\left(n \mu-1, w_{1}(\underline{\alpha})\right), \quad n \mu>1,
$$

for which

$$
\gamma^{k}(\tau, z)=\gamma\left(\tau, z / a_{1}\right)-\gamma\left(\tau, z / a_{2}\right),
$$

and $\gamma(.,$.$) denotes the wel1-known incomplete gamma function (Andrews [9]);$

(iii)

$$
\pi(\sigma)=C^{-1}(n \mu, \underline{\alpha}, \lambda) \underset{\underline{1}}{\sum} \exp \left[-\left\{\sigma / \lambda+w_{\underline{1}}(\underline{\alpha}) / \sigma\right\}\right] \quad \sigma^{-n \mu},
$$

where

$$
C(n \mu, \underline{\alpha}, \lambda)=\lambda^{-(n \mu-1) / 2} \sum_{\underline{i}}\left[w_{\underline{1}}(\underline{\alpha})\right]^{-(n \mu-1) / 2} k_{n \mu-1}\left(2\left[w_{\underline{1}}(\underline{\alpha}) / \lambda\right]^{1 / 2}\right),
$$

and $\mathrm{K}_{\tau}(\cdot)$ is the modified Bessel function of the third kind of order $\tau$ (Andrews [9]); (iv)

where

$$
\pi(\sigma)=D^{-1}(n \mu, \underline{\alpha}, c) \sum_{\underline{1}}^{\sum} \exp \left[-w_{\underline{1}}(\underline{\alpha}) / \sigma\right] \sigma^{-(n \mu+c)},
$$

$$
D(n \mu, \underline{\alpha}, c)=\Gamma(n \mu+c-1) \underset{\underline{1}}{\sum\left[w_{\underline{1}}(\underline{\alpha})\right]^{-(n \mu+c-1)}, n \mu+c>1 .}
$$

From the above posterior densities, the posterior means of $\sigma$, also respectively, are:

where

$$
\sigma^{*}=\sum_{\underline{1}} A_{1}^{*}(\underline{\alpha}) \frac{a+w_{1}(\underline{\alpha})}{n \mu+b-1}, n \mu+b>1,
$$

$$
A_{\underline{1}}^{*}(\underline{\alpha})=A^{-1}(n \mu, \underline{\alpha}, a, b) \Gamma(n \mu+b)\left[a+w_{\underline{1}}(\underline{\alpha})\right]^{-(n \mu+b)} \text {; }
$$

$$
\sigma^{*}=B^{-1}\left(n \mu, \underline{\alpha}_{1} a_{1}, a_{2}\right) \quad B\left(n \mu-1, \underline{\alpha}, a_{1}, a_{2}\right), \quad n \mu>1,
$$

$$
\sigma^{*}=c^{-1}(n \mu, \underline{\alpha}, \lambda) \quad C(n \mu-1, \underline{\alpha}, \lambda),
$$

(iv)

where

$$
\begin{aligned}
& \sigma^{*}=\sum_{\underline{1}} D_{\underline{1}}^{*}(\underline{\alpha}) \frac{w_{1}(\underline{\alpha})}{n \mu+c-2}, \quad n \mu+c>2, \\
& D_{\underline{1}}^{*}(\underline{\alpha})=D^{-1}(n \mu, \underline{\alpha}, c) \Gamma(n \mu+c-1)\left[w_{\underline{1}}(\underline{\alpha})\right]^{-(n \mu+c-1)} ;
\end{aligned}
$$

and the posterior variances of $\sigma$ are:

$$
\operatorname{Var}(\sigma)=\sum_{\underline{1}} A_{\underline{1}}^{\star 2}(\underline{\alpha}) \frac{\left[a+w_{1}(\underline{\alpha})\right]^{2}}{(n \mu+b-1)^{2}(n \mu+b-2)}, \quad n \mu+b>2,
$$

(ii)

$$
\operatorname{Var}(\sigma)=\frac{B\left(n \mu, \underline{\alpha}, a_{1}, a_{2}\right) B\left(n \mu-2, \underline{\alpha}, a_{1}, a_{2}\right)-B^{2}\left(n \mu-1, \underline{\alpha}, a_{1}, a_{2}\right)}{B^{2}\left(n \mu, \underline{\alpha}, a_{1}, a_{2}\right)},
$$


provided $n \mu>2$;

(iii)

(iv)

$$
\operatorname{Var}(\sigma)=\frac{C(n \mu, \underline{\alpha}, \lambda) c(n \mu-2, \underline{\alpha}, \lambda)-c^{2}(n \mu-1, \underline{\alpha}, \lambda)}{c^{2}(n \mu, \underline{\alpha}, \lambda)},
$$

$$
\operatorname{Var}(\sigma)=\sum_{1} D_{\underline{i}}^{*}(\underline{\alpha}) \frac{w_{1}^{2}(\underline{\alpha})}{(n \mu+c-2)^{2}(n \mu+c-3)}, \quad n \mu-c>3 .
$$

3. ESTIMATION OF $\sigma$ WHEN $\mu$ IS A RANDOM VARIABLE

When both $\mu$ and $\sigma$ are random variables having the mixture prior distributions given by (1.6) and (1.6a), the likelihood function in this case must satisfy the following relation:

$$
L(\mu, \sigma) \propto \frac{u^{\mu-1}}{\Gamma^{n}(\mu)} \underline{i} \exp \left[-w_{\underline{i}}(\underline{\alpha}) / \sigma\right] \sigma^{-n \mu}
$$

where $u=\pi_{i=1}^{n} x_{i}$. This implies that the marginal posterior probability distribution of $\mu$ is given by

$$
p_{j}^{*}(\underline{\alpha})=\frac{p_{j}^{\prime}(\underline{\alpha})}{\sum_{s=1}^{m} p_{s}^{\prime}(\underline{\alpha})}, \quad j=1,2, \ldots, m .
$$

where

$$
p_{j}^{\prime}(\underline{\alpha})=p_{j} u^{\mu_{j}-1} \frac{\Gamma\left(n \mu_{j}+\nu_{j}\right)}{\Gamma^{n}\left(\mu_{j}\right)} \sum_{\underline{i}}\left[w_{\underline{i}}(\underline{\alpha})+\mu_{j}\right]^{-\left(n \mu_{j}+v_{j}\right)} .
$$

Also, the conditional posterior density of $\sigma$ given $\mu^{=} \mu_{j}$ is given by

$$
h\left(\sigma \mid \mu=\mu_{j}\right)=Q^{-1}\left(\underline{\alpha}, \mu_{j}, \nu_{j}\right) \underset{\underline{i}}{\sum} \exp \left[-\left\{w_{\underline{i}}(\underline{\alpha})+\mu_{j}\right\} / \sigma\right] \sigma^{-\left(n \mu_{j}+\nu_{j}+1\right)},
$$

where

$$
Q\left(\underline{\alpha}, \mu_{j}, \nu_{j}\right)=\Gamma\left(n \mu_{j}+\nu_{j}\right) \quad \sum_{\underline{1}}\left[w_{\underline{i}}(\underline{\alpha})+\mu_{j}\right]-\left(n \mu_{j}+\nu_{j}\right) .
$$

Now, the product of (3.2) and (3.4) yields the joint posterior distribution of $\mu$ and $\sigma$. The (unconditional) posterior mean of $\sigma$ is of the form

$$
\sigma^{*}=\sum_{\underline{i}} \sum_{j=1}^{m} Q_{\underline{i}, j}^{*}(\underline{\alpha}) \frac{w_{\underline{1}}(\alpha)+\mu_{j}}{n \mu_{j}+\nu_{j}-1}, \quad n \mu_{j}+\nu_{j}>1,
$$

where

$$
Q{ }^{*}{ }_{i, j}(\underline{\alpha})=p_{j}^{*}(\underline{\alpha}) Q^{-1}\left(\underline{\alpha}, \mu_{j}, \nu_{j}\right) \Gamma\left(n \mu_{j}+v_{j}\right)\left[w_{\underline{i}}(\underline{\alpha})+\mu_{j}\right]^{-\left(n \mu_{j}+\nu_{j}\right)},
$$
provided $n \mu_{j}+v_{j}>1$ for all $j=1,2, \ldots, m$; and the posterior variance is

$$
\operatorname{Var}(\sigma)=\sum_{\underline{i}} \sum_{j=1}^{m} Q_{\underline{i}, j}^{* 2}(\underline{\alpha}) \frac{\left[w_{1}(\underline{\alpha})+\mu_{j}\right]^{2}}{\left(n \mu_{j}+v_{j}-1\right)^{2}\left(n \mu_{j}+v_{j}-2\right)},
$$


provided $n \mu_{j}+v_{j}>2$ for all $j=1,2, \ldots, m$.

4. HOMOGENEOUS CASE WHEN $\mu$ IS FIXED

In the case of simple random sample from a complete life test, the posterior mean, and variance of $\sigma$ can be derived from those in section 2 by letting $\alpha_{r}=1$, for a $11 \mathrm{r}=1,2, \ldots, \mathrm{k}$.

Respectively considering the priors (1.2), (1.3), (1.4) and (1.5), the posterior means of $\sigma$, in the homogeneous case, are:

(i)

$$
\begin{aligned}
& \qquad \sigma^{*}=\frac{\alpha+n \bar{x}}{n \mu+b-1}, \quad n \mu+b>1, \\
& \text { where } \bar{x} \text { is the sample mean; }
\end{aligned}
$$

$$
\sigma^{*}=\frac{r^{*}(n \mu-2, n \bar{x})}{r^{*}(n \mu-1, \bar{n} \bar{x})} n \vec{n}, \quad n \mu>2
$$

$$
\sigma^{*}=\frac{\bar{n} \bar{x}}{n \mu+c-2}, \quad n \mu+c>2,
$$

and the posterior variances are:

(1)

$$
\operatorname{Var}(\sigma)=\frac{(a+n \bar{x})^{2}}{(n \mu+b-1)^{2}(n \mu+b-2)}, \quad n \mu+b>2,
$$

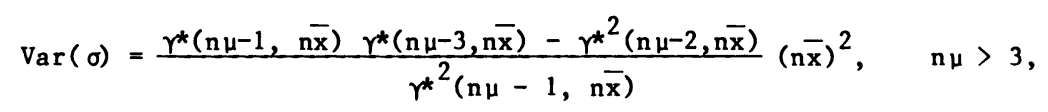

(i1i)

$$
\operatorname{Var}(\sigma)=\frac{k_{n \mu-1}\left(2[n \bar{x} / \lambda]^{1 / 2}\right) k_{n \mu-3}\left(\overline{2}[n x / \lambda]^{1 / 2}\right)-k_{n \mu-2}\left(2[\overline{n x} / \lambda]^{1 / 2}\right)}{k_{n \mu-2}^{2}\left(2[n \bar{x} / \lambda]^{1 / 2}\right)} \lambda n \bar{x},
$$

(iv)

$$
\operatorname{Var}(\sigma)=\frac{(n \bar{x})^{2}}{(n \mu+c-2)^{2}(n \mu+c-3)}, \quad n \mu+c>3
$$

The above results agree with Bhattacharya [6], Canavos and Tsokos [7], and Lwin and Singh [8].

\section{HOMOGENEOUS CASE WHEN $\mu$ IS A RANDOM VARIABLE}

When $\mu$ and $\sigma$ have the mixture distribution given by $(1.6)$ and $(1.6 a)$, the posterior mean of $\sigma$ in this case is

$$
\sigma^{*}=\sum_{j=1}^{m} p_{j}^{*} \frac{n \bar{x}+\mu_{j}}{n \mu_{j}+\nu_{j}-1}, \quad n \mu_{j}+\nu_{j}>1,
$$


where

$$
p_{i}^{*}=\frac{p_{j}^{\prime}}{\sum_{s=1}^{m} p_{s}^{\prime}}, \quad j=1,2, \ldots, m
$$

with

$$
p_{j}^{\prime}=p_{j} u^{\mu_{j}-1} \frac{\Gamma\left(n \mu_{j}+v_{j}\right)}{\Gamma^{n}\left(\mu_{j}\right)\left(n \bar{x}+n \mu_{j}\right)^{n \mu_{j}+v_{j}}}
$$

and the posterlor variance of $\sigma$ takes the form

$$
\operatorname{Var}(\sigma)=\sum_{j=1}^{m} p_{j}^{* 2} \frac{\left(n \bar{x}+\mu_{j}\right)^{2}}{\left(n \mu_{j}+\nu_{j}-1\right)^{2}\left(n \mu_{j}+\nu_{j}-2\right)}, \quad n \mu_{j}+\nu_{j}>2 .
$$

These results agree with Lwin and Singh [8], and Martz and Waller [10].

ACKNOWLEDGEMENT. I would like to express my thanks to L. Ringuette and the referee for their valuable comments and suggestions for improving the presentation of this paper.

\section{REFERENCES}

1. BARNETT, V. and LEWIS, T., Outliers in Statistical Data, Second Edition, John Wiley \& Sons, 1978.

2. SINHA, S.K., Life Testing and Reliability Estimation for Non-Homogeneous Data a Bayesian Approach, Comm. Statist. A-Theory Methods 2(3) (1973) 235-243.

3. LINGAPPAIAH, G.S., Effect of Outliers of the Estimation of Parameters, Metrika 23(1976) 27-30.

4. LINGAPPAIAH, G.S., Problem of Estimation when the Outliers are Present, Trabajos. Estadist. Investigacion Oper. 30(3)(1979) 71-80.

5. RAIFFA, H., Decision Analysis, MA:Addison-Wesley, 1968.

6. BHATTACHARYA, S.K., Bayesian Approach to Life Testing and Reliability Estimation, J. Amer. Statist. Assoc. 26(1976) 48-62.

7. CANAVOS, G.C. and TSOKOS, C.P., A Study of an Ordinary and Empirical Bayes Approach to Reliability Estimation in the Gamma Life Testing Model, Proceedings 1971 Annual Reliability and Maintainability Symposium, (1971), 343-349.

8. LWIN, T. and SINGH, N., Bayesian Analysis of the Gamma Model in Reliability Estimation, IEEE Trans. Reliability R-23(5), (1974), 314-319.

9. ANDREWS, L.C., Special Functions for Engineers and Applied Mathematicians, Macmillan Publishing Company, 1985.

10. MARTZ, H.F. and WALLER, R.A., Bayesian Reliability Analysis, John Wiley \& Sons, 1982. 


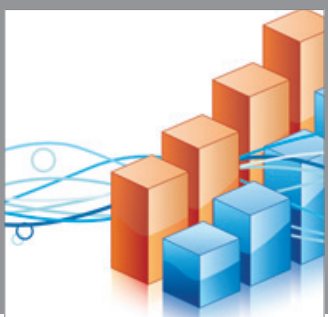

Advances in

Operations Research

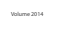

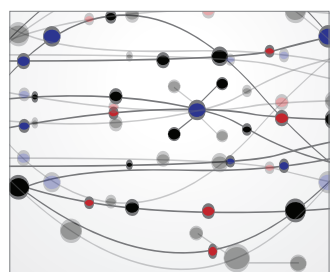

\section{The Scientific} World Journal
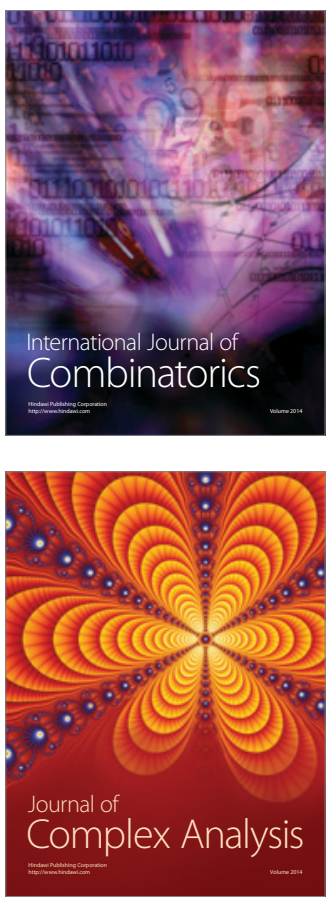

International Journal of

Mathematics and

Mathematical

Sciences
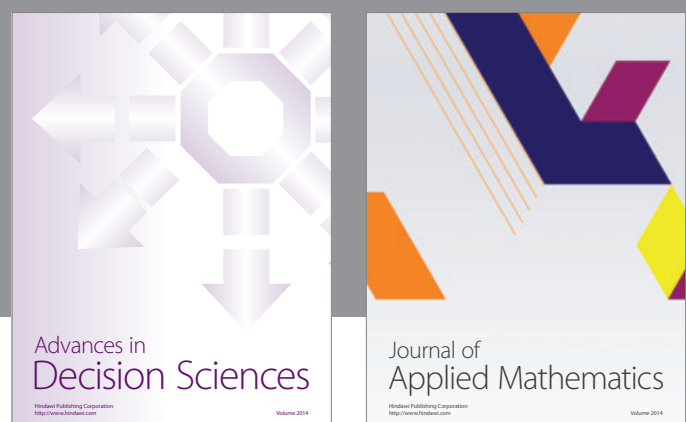

Journal of

Applied Mathematics
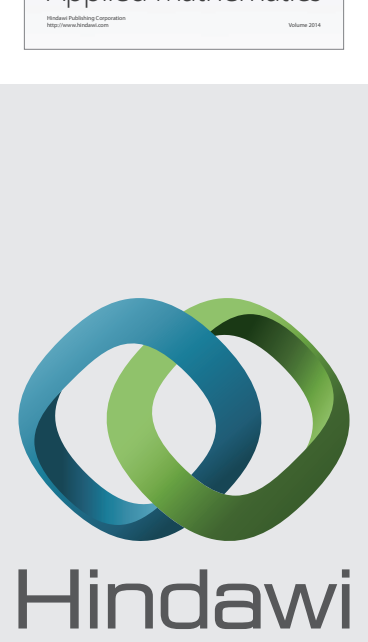

Submit your manuscripts at http://www.hindawi.com
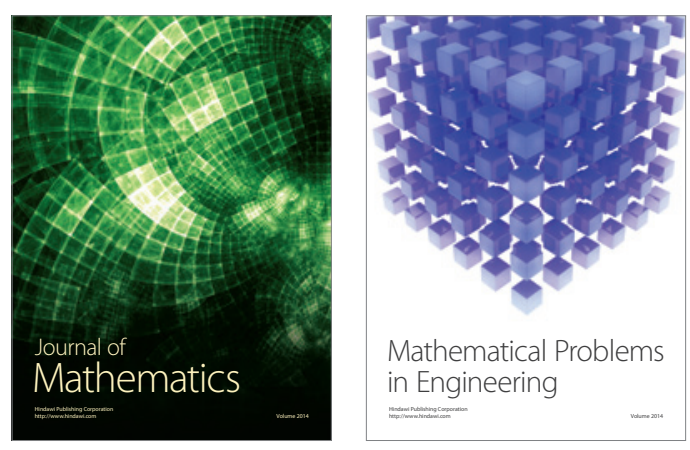

Mathematical Problems in Engineering
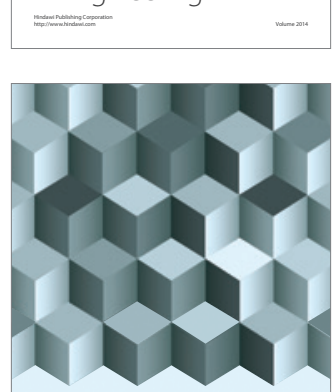

Journal of

Function Spaces
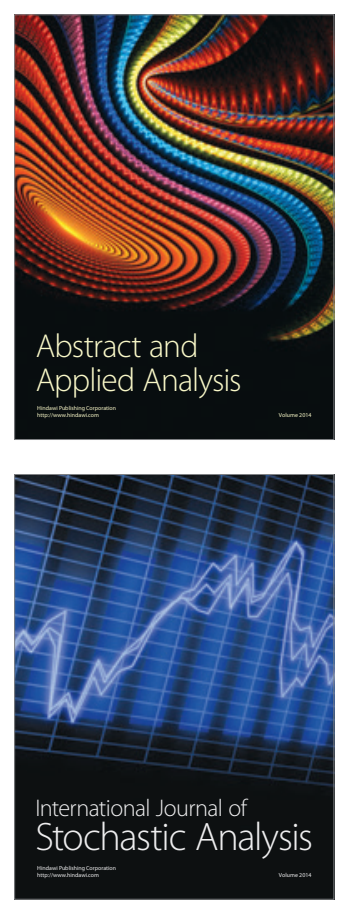

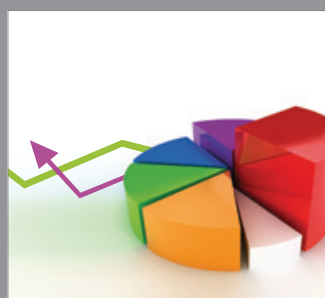

ournal of

Probability and Statistics

Promensencen
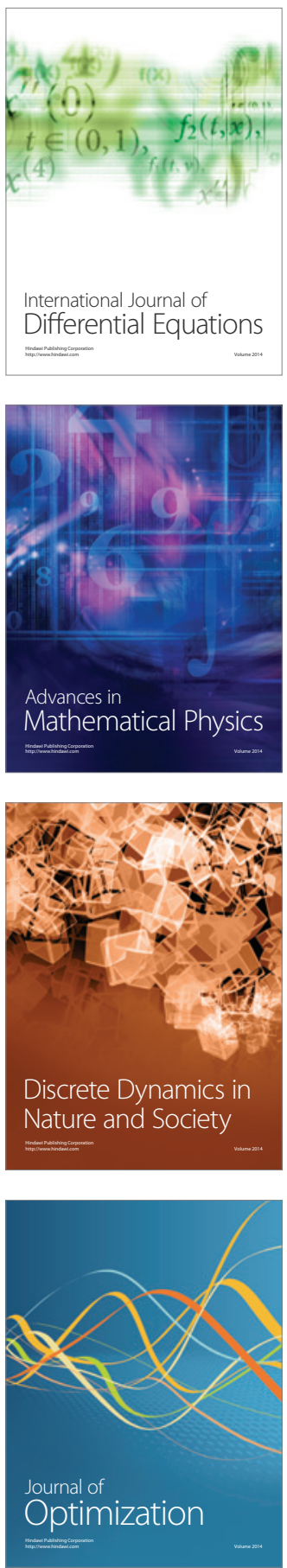